\title{
Design of Electric Power Steering for Forklift Based on ARM
}

\author{
Ma Dongmei \\ Changzhou University \\ Changzhou, China \\ mdm@cczu.edu.cn
}

\begin{abstract}
After analyzing Electric Power Steering (EPS) and electric control unit (ECU), a controller system based on ARM LPC1752 is designed. Direction control circuit, H-bridge control circuit and PWM technology were used to control a motor. The PD fuzzy control algorithm was used to control the steering system too. The system has got through the test of relevant electrical properties. Practical use shows that the system has good control performance.
\end{abstract}

Keywords-ARM controller; H-bridge control circuit; PD control; Over-current protection

\section{INTRODUCTION}

The Electric Power Steering (EPS) is a kind of power steering that depends on motor to provide auxiliary torque. Compared with the traditional Hydraulic Power Steering, the EPS is easier to adjust and much more flexible to assemble and can provide power on more conditions. It can save energy and protect the environments. It's the development direction of the Power Steering ${ }^{[1]}{ }^{[3]}$. In this paper, an EPS system based on ARM is designed after analyzing the principle of EPS and electric control unit (ECU). The PD fuzzy control algorithm was used to control the steering system too. The system has got through the test of relevant electrical properties and has been proved to have excellent assist characteristic.

\section{Working Process of ForkLIFT Electric Power STEERING}

When the electric forklift steering wheel rotates, rotation direction sensor outputs an analog signal to ECU controller. The analog signal is corresponds to the steering wheel's rotation angle. ECU combines this rotation angle signal and the speed signal coming from speed sensor and than determines a target PWM to control the motor rotation direction and speed [2]. The motor's torque output is delivered to steering output shaft through reducing mechanism. And then makes the steering wheel rotate certain angle with the use of steering gear ${ }^{[4]}$. The process realized the power function to the steering system.

\section{STRUCTURE AND PRINCIPLE OF The CONTROLleR}

A/D acquisition circuit, H-bridge motor control circuit and protection circuit compose the controller, as shown in Figure 1. The controller collects torque signal and speed signal and then transmits them to ARM chip. According to the control rules, the system determines the motor's rotation direction and speed to drive the motor through $\mathrm{H}$-bridge circuit by the mode of PWM. At the same time, the system takes sample of the motor's current to control the motor or protect the system. When the motor current is greater than the over-current set value, or the speed is greater than the set value, in order to protect the security of the system the controller will send a signal to the relay to cutoff the motor's power. When the system works normally, the energy will be supplied again.

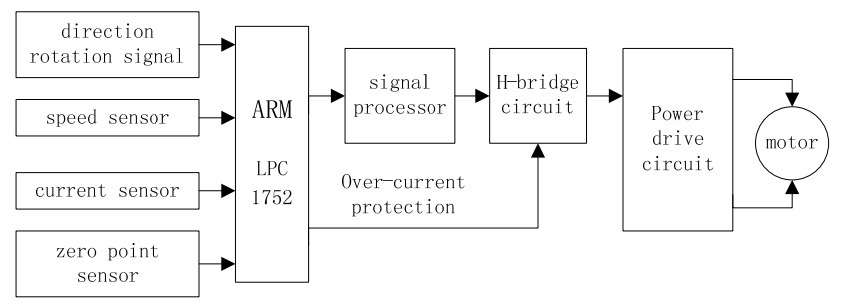

Figure 1. Structure of the controller.

\section{DESIGN OF CONTROLleR}

ARM LPC1752 is the CPU of the controller. ARM LPC1752 has abundant hardware resources. So, the circuit is simplified, the reliability is improved, and the system will be easier to be extended and upgraded. Direction control signal and PWM signal are loaded to H-bridge driver circuit to control the motor. The use of HIP4082 avoids the possibility direct connection of the MOSFETs at the same time. Pulse width modulation mode uses single polarity PWM. The duty ratio of PWM is easier to be adjusted and the system is much more reliable.

\section{A. ARMLPC1752 microprocessor}

The LPC1752 is ARM Cortex-M3 based microcontroller for embedded applications featuring a high level of integration and low power consumption. The peripheral complement of the LPC1752 includes up to $512 \mathrm{kB}$ of flash memory, up to $64 \mathrm{kB}$ of data memory, Ethernet MAC, USB Device/Host/OTG interface, 8-channel general purpose DMA controller, 4 UARTs, 2 CAN channels, 2 SSP controllers, SPI interface, 3 I2C-bus interfaces, 2-input plus 2-output I2S-bus interface, 6 channel 12-bit ADC, 10-bit DAC, motor control PWM, Quadrature Encoder interface, 4 general purpose timers, 6-output general purpose PWM, ultra-low power Real-Time Clock (RTC) with separate battery supply, and up to 52 general purpose I/O pins. At the same time ARM LPC1752 supports C language, this is helpful for the software's development and debugging of the control system. In the design of this system, in addition to 
the corresponding memory and timer functions, the DAC and PWM are mainly used.

\section{B. Controller pins function description}

The center controller's input and output pins are indicated in fiigure 2.

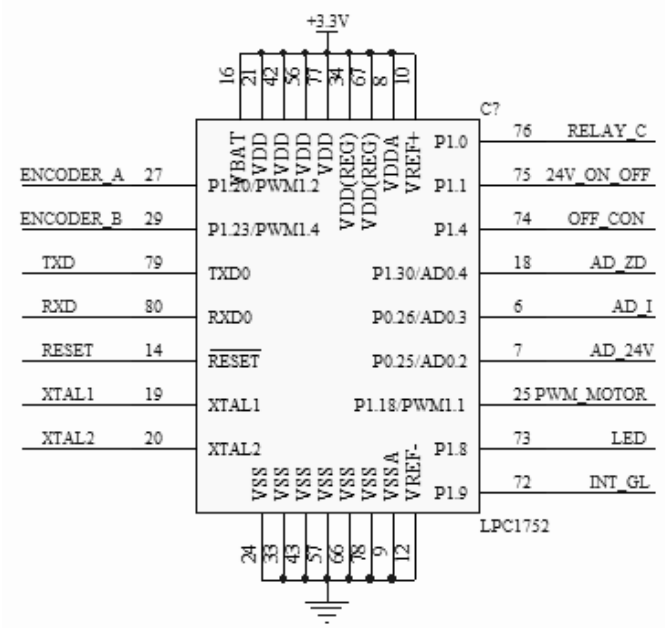

Figure 2. Input and output pins of LPC1752.

The expatiation of the pins flows below.

- RESET: Reset signal input suplied by chip CS8363. At the same time, chip CS8363 suplies 3.3V voltage to ARM processor.

- XTAL1, XTAL2: Work clock signal input. The working frequency of the system is $12 \mathrm{MHz}$.

- $\quad$ RXD, TXD: RXD and TXD are connected with chip RS232, used for communication with computer. It is used to download a program and display necessary information on the PC screen.

- ENCODER_A, ENCODER_B: Speed encoder signal input pins. Interrupt mode is used to read forklift steering motor rotation speed.

- 24V_ON_OFF: The $24 \mathrm{~V}$ power control signal output. It is used to open or close the $24 \mathrm{~V}$ power.

- PWM_MOTOR: Motor speed control PWM signal output, used to control the steering motor's speed and direction.

- RELAY_C: Motor power's relay control signal, according to self state to decide whether to provide power to the motor.

- OFF_CON: The recovery output signal of over current protection. When over-current happens, PWM signal output is prohibited. After a period of time's delay, PWM signal output is allowed again to determine whether over-current exists.

- INT GL: Over-current input interrupt application signal. When over-current happens, its edge produce interrupt request and the system makes corresponding processing in time.

- LED: LED lamp is used for indicating the running status of the controller and the causes of the failures.
- AD_ZD: The rotation angle's encoder input signal, the size of the voltage reflects the angle size of the forklift steering wheel rotates.

- AD_I: Motor driver current input. It is used to judge $\mathrm{r}$ the motor is over-current or not.

- AD_24V: The input of supply voltage, reflects the size of the power supply voltage. When the power is too big or too small, the system will output alarm signal and then stop working.

\section{A/D data sampling}

LPC1752 uses 12 successive approximation ADC type. Input voltage range is $0 \sim 3.0 \mathrm{~V}$, and conversion distinguish ability is $2.9 \mathrm{mV}$. For torque sensor, the output voltage range is $1 \sim 4 \mathrm{~V}$, so the signal only needs low pass filtering processing and partial pressure processing. To the motor sampling current, with positive and negative difference, still needs level conversion to make it into positive voltage. The AD_I signal as shown in figure 5 is this positive voltage.

\section{Motor control circuit}

The motor control circuit is composed of direction control circuit and H-bridge motor control circuit. The motor control circuit diagram is shown in Figure 3.

The PWM signal comes from ARM passes through $74 \mathrm{HC} 00$ chip, and then generates two signals which have same duty ratio and opposite polarity. These two signals are transmitted to ALI and BLI of HIP4082 chip.

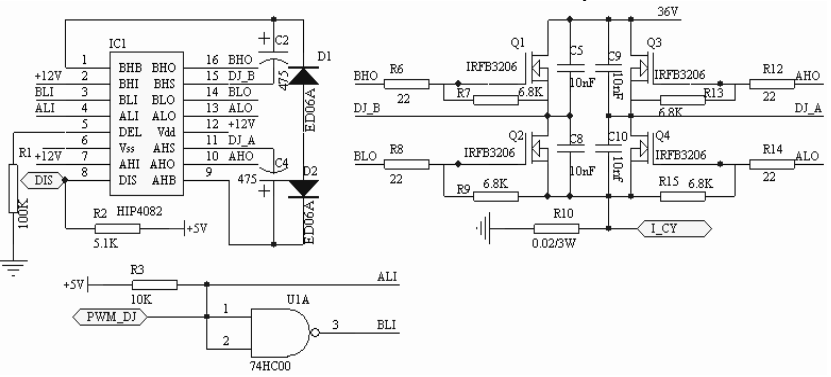

Figure 3. Motor control circuit diagram.

DIS is the input signal of over-current protection, it is in low level normally. When DIS is in high level, the four output signals $\mathrm{AHO}, \mathrm{BHO}, \mathrm{ALO}$ and BLO are all in low level. The four MOSFETs Q1,Q2,Q3 and Q4 are turned off and the over-current protection is realized. The DEL pin of HIP4082 is connected to ground through a resistance. The resistance plays a role of delay of the up-down tubes' conducting conversion. It makes the up-down MOSFETS are not conducted at the same time. This improves the system's reliability greatly. The oscillogram of signal to drive motor is demonstrated in Figure 4. 


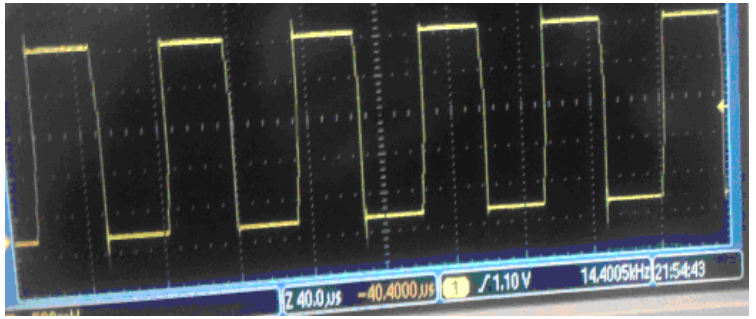

Figure 4. Motor control circuit diagram.

\section{E. Analysis of control process}

When the duty ratio of PWM signal is equal to $50 \%$, the turn-on time of Q1 and Q4, Q2 and Q3 is same, and the forward and reverse signal time on the motor's two sides is same too. So the two sides' power is same and the motor keeps stationary. If the duty ratio of PWM signal is greater than $50 \%$, the turn-on time of Q1 and Q4 is greater than Q2 and Q3. The power on forward direction is greater than the reverse side. The motor rotates forward. If the duty ratio of PWM signal is less than $50 \%$, the turn-on time of Q1 and Q4 is less than Q2 and Q3. The power on forward direction is less than the reverse side. The motor rotates in reverse direction.

When forklift steering wheel rotate, it drives the sensor rotates, and outputs the rotation angle analog signal at the same time. When the steering wheel rotates $0 \sim 180$ degrees, the corresponding sensor output $1 \sim 3.5 \mathrm{~V}$ voltage. After the partial pressure processing, the voltage is added to $A / D$ channel of ARM chip. ARM converts the voltage signal into corresponding digital quantity, and then converts the digital quantity into corresponding rotation angle to control the PWM duty ratio, i.e., to control motor rotation direction and rotation speed. When motor rotates, the coding sensor connected with it outputs pulse signal, which reflects the motor rotation speed and angle. The coding sensor signal is transmitted to corresponding input pin of LPC1752, and the program can read the value and direction.

The program compares the wheel rotation angle $\omega 1$ and the angle value $\omega 2$ read by coding sensor. If $\omega 1=\omega 2$, it shows that the forklift's rotation angle and direction are according with the steering's angle and direction. And then the motor stop turning. When $\omega 1>\omega 2$, it indicates that the forklift's rotation angle do not reach the wheel positive direction angle. The motor continues to rotate in positive direction until $\omega 1=\omega 2$. When $\omega 1<\omega 2$, it indicates that the forklift's rotation angle do not reach the wheel reverse direction angle. The motor continues to rotate in reverse direction until $\omega 1=\omega 2$.

\section{F. Design of over-current protection circuit}

Over-current protection circuit is shown in figure 5 . Access a current sampling resistance R10 between the MOS tubes and ground. The value of R10 is $20 \mathrm{~m} \Omega$, the motor protection current value takes $10 \mathrm{~A}$. Then the maximum voltage of $\mathrm{R} 10$ is $0.2 \mathrm{~V} . \mathrm{C}_{24}$ and $\mathrm{R}_{24}$ compose filter circuit. The filter circuit converses pulse voltage into direct voltage. The operational amplifier U7A amplify this DC and output the amplifying signal to the reverse input pin of U7B. The positive input reference voltage is $4 \mathrm{~V}$. Normally, the reverse input voltage is less than the positive voltage. The U7B output voltage is high level. Chip $74 \mathrm{HC} 00$ changes it into low level, and the value of DIS is 0 . So the H-bridge control circuit works normally. When motor current is greater than $10 \mathrm{~A}$, the amplifying output signal of U7A will be greater than $4 \mathrm{~V}$. The reverse input voltage is greater than the positive voltage. The U7B output voltage is low level. Chip $74 \mathrm{HC} 00$ changes it into high, and the value of DIS is 1 . The H-bridge control circuit stops working to turn off the power of the motor. So the system is well protected.

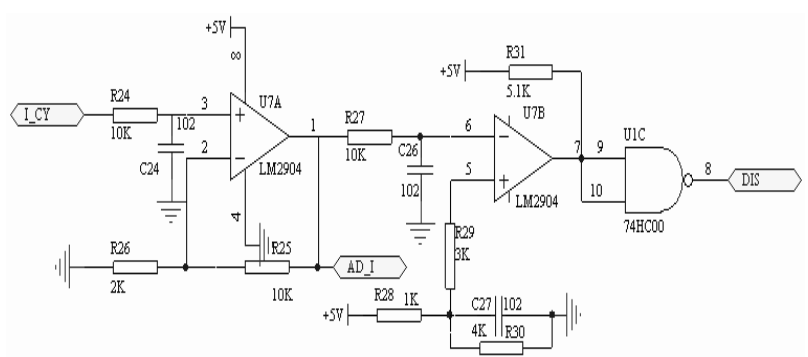

Figure 5. Over-current protection circuit.

\section{G. Software design}

The software program is written in Keil uVision4. Software control flow chart is shown in figure 6. The main program is composed of initialization portion and main loop program. Serial port, timer, external interrupt, ADC and PWM are initialized in the initializer. In the loop program, the control to the motor and over-current are realized.

As a time reference, $\mathrm{T} 0$ is a $0.1 \mathrm{mS}$ timer in order to determine the motor rotation speed. The external interrupt 0 counts to the motor encoder's input pulse to determine the forklift rotation angle. ADC converts the analog signal of rotation direction and motor current to corresponding digital quantity. Then the digital quantity is converted to corresponding rotation angle and motor current value. RS232 serial outputs current running state. PC machine receives and displays relevant information. PWM signal is the direction control signal and speed control signal of the motor. The motor current rotation speed and motor current value are transmitted into PD controller. According to the steering wheel rotation signal, ARM processor output a corresponding PWM value to control the rotation direction and rotation speed of motor. So, the system has good tracking effect. 


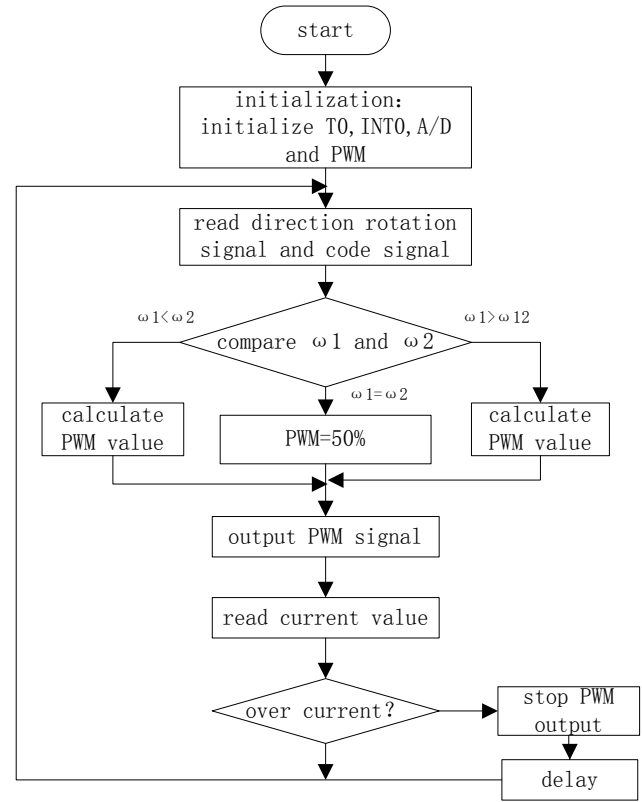

Figure 6. Software control flow chart.

\section{SYSTEM CONTROL STRATEGY AND IMPLEMENTATION}

Domestic and overseas scholars research different kinds of EPS control strategies, such as PID control ${ }^{[5][6]}$, H-robust control $^{[7][8]}$, fuzzy control, etc. Because the torque signal and speed signal is very suitable for fuzzy control, and PD control has better control performance. We adopt the PD fuzzy control strategy. System control strategy chart is shown in figure 7.

Direction rotation angle signal is the input of the PD controller. $\mathrm{Kp}$ is the scale factor and $\mathrm{Kd}$ is the derivative coefficient of PD. PD controller collects the signal of torque sensor, than sets $\mathrm{Kp}$ and $\mathrm{Kd}$ parameters online to determine the system's target value. The use of the samples shows that motor's angle speed has good tracking performance to the input signal of steering wheel with the use of PD controller.

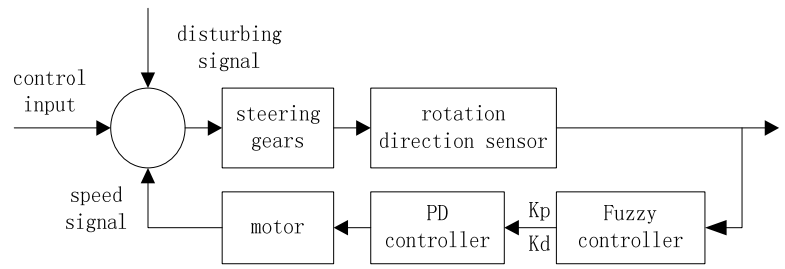

Figure 7. System control strategy chart.

\section{CONCLUSION}

The actual control circuit board is shown in figure 8 . The following conclusions are concluded.

- ARM processor is used in this system, which has high speed and flexible control algorithm. The use of ARM simplifies the system's circuit and improves the reliability and the system will be easier to be extended and upgraded.
- $\quad$ Using H-bridge drive chip HIP4082 to drive power tubes, this makes the system have higher efficiency and lower heat.

- The use of PD fuzzy control strategy improves the tracking performance.

- The software process is reasonable, making the system run stably and reliably.

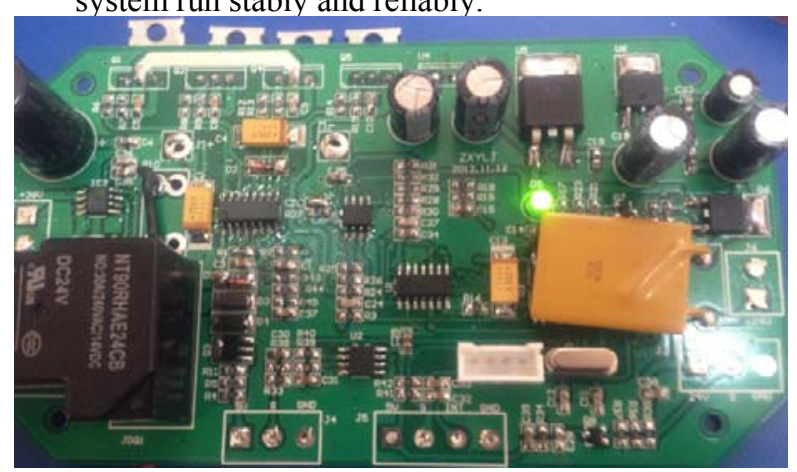

Figure 8. Actual control circuit board.

The system has got through the test of relevant electrical properties. Up to now, the samples have been used for half a year. Practical application shows that the system has good rotation tracking performance. It is stable and reliable. Controller performance has reached the users' requirements.

\section{REFERENCES}

[1] Ding Junqiang, Tang Lan, Wang Yuanyuan, Xie Lin, "Simulated research on assistance control strategy of electric power streeing system," Journal of Chengdu University(Natural Science Edition), Chengdu, Sicuan, vol,30, no.1, Mar.2011, pp.52 54.

[2] Yang Xinhua, Wang Yongshuang, "Development of electric power steering system based on DSP," Modern Electronics Technique, Xian, Shan Xi, vol.34, no.11, June, 2011, pp.208 210.

[3] Liu L, Nagai M, On torque control of vehicle handling and steering feel for avoidance maneuver with electric power steering [C], Proceedings of the 17th World Congress, The International Federation of Automatic Control, Seoul, Korea: IFAC Press, 2008, pp.12703 12708.

[4] M.Bland, J.S. Thomsen, "Electrical steering of vehicles fault tolerant analysis and design," Microelectronics Reliability, 2006, pp.1421 1432.

[5] Kim, J, Song J. Control logic for an electric power steering system using assistmotor[J]. Mechatronics, December 2002, pp.447 459.

[6] Jeonghoon S, Kwanguck B, Heung Seob Kim. Model devel-opment and control methodology of a new electric power steering system[J]. Poc.Instn Mech.Engrs Part D:J. Automo-bile Engineering,2004, pp.68-73.

[7] Qiu Ming, Yang Jiajun, Liu Zhao and Ye Geng, "Steering system of electric power assist base on $\mathrm{H} \infty$ Robust control principle," Journal of Huazhong University of Science and Technology, Hubei, Wuhan,vol.32, vo.12, pp.71 73, December 2002.

[8] Li Shulong, Xu Chao, "Fuzzy control in electric power assisted streeing system," Journal of highway and transportation research and development, vol.21, no.4, pp.101 103, April,2004. 